Acta Crystallographica Section E

Structure Reports

Online

ISSN 1600-5368

\section{2-Iminiumyl-1,3-diazepane-4- carboxylate}

\section{Feng Yang}

School of Chemistry and Chemical Engneering, Guangxi Normal University, Guilin 541004, People's Republic of China

Correspondence e-mail: jxyangfeng@gmail.com

Received 20 October 2010; accepted 28 November 2010

Key indicators: single-crystal X-ray study; $T=293 \mathrm{~K}$; mean $\sigma(\mathrm{C}-\mathrm{C})=0.005 \AA$; $R$ factor $=0.042 ; w R$ factor $=0.116$; data-to-parameter ratio $=8.3$.

The title compound, $\mathrm{C}_{6} \mathrm{H}_{11} \mathrm{~N}_{3} \mathrm{O}_{2}$, is a cyclized derivative of $\mathrm{L}$ arginine and the molecule is a zwitterion with the positive and negative charge residing in the guanidinium and carboxylate groups, respectively. The conformation of 1,3-diazepane ring is close to a twisted chair. One intramolecular and three intermolecular $\mathrm{N}-\mathrm{H} \cdots \mathrm{O}$ hydrogen bonds stabilize the molecular conformation and the crystal structure, respectively.

\section{Related literature}

For related structures, see: Karapetyan $(2008 a, b)$.

\section{Experimental}

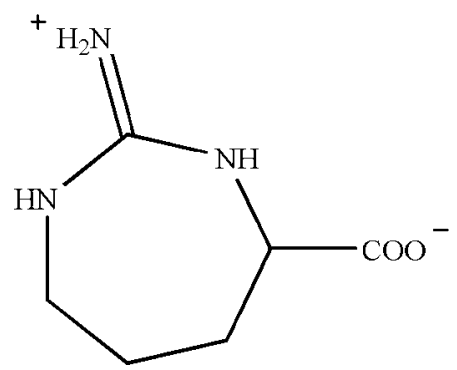

Crystal data

$\mathrm{C}_{6} \mathrm{H}_{11} \mathrm{~N}_{3} \mathrm{O}_{2}$

$M_{r}=157.18$

Orthorhombic, $P 2_{1} 2_{1} 2_{1}$

$$
\begin{aligned}
& b=8.7979(5) \AA \\
& c=14.2036(7) \AA \\
& V=771.51(7) \AA^{3} \\
& Z=4
\end{aligned}
$$

$$
\begin{aligned}
& \text { Mo } K \alpha \text { radiation } \\
& \mu=0.10 \mathrm{~mm}^{-1} \\
& T=293 \mathrm{~K} \\
& 0.23 \times 0.15 \times 0.10 \mathrm{~mm}
\end{aligned}
$$

\section{Data collection}

Siemens SMART CCD areadetector diffractometer

Absorption correction: multi-scan (SADABS; Sheldrick, 1996)

$T_{\min }=0.773, T_{\max }=1.000$

\section{Refinement}

$R\left[F^{2}>2 \sigma\left(F^{2}\right)\right]=0.042$

$w R\left(F^{2}\right)=0.116$

$S=1.08$

834 reflections
3426 measured reflections 834 independent reflections 694 reflections with $I>2 \sigma(I)$ $R_{\text {int }}=0.026$

100 parameters

$\mathrm{H}$-atom parameters constrained

$\Delta \rho_{\max }=0.25{\mathrm{e} \AA^{-3}}^{-3}$

$\Delta \rho_{\min }=-0.23{\mathrm{e} \AA^{-3}}^{-3}$

Table 1

Hydrogen-bond geometry $\left(\AA,{ }^{\circ}\right)$.

\begin{tabular}{lllll}
\hline$D-\mathrm{H} \cdots A$ & $D-\mathrm{H}$ & $\mathrm{H} \cdots A$ & $D \cdots A$ & $D-\mathrm{H} \cdots A$ \\
\hline $\mathrm{N} 1-\mathrm{H} 1 A \cdots \mathrm{O} 1^{\mathrm{i}}$ & 0.86 & 2.17 & $2.918(3)$ & 146 \\
$\mathrm{~N} 3-\mathrm{H} 3 A \cdots \mathrm{O} 2^{\mathrm{i}}$ & 0.86 & 2.06 & $2.870(4)$ & 157 \\
$\mathrm{~N} 3-\mathrm{H} 3 B \cdots \mathrm{O} 2^{\mathrm{ii}}$ & 0.86 & 1.95 & $2.788(4)$ & 163 \\
$\mathrm{~N} 2-\mathrm{H} 2 A \cdots \mathrm{O} 1$ & 0.86 & 2.19 & $2.601(3)$ & 109
\end{tabular}

Symmetry codes: (i) $-x+\frac{3}{2},-y+1, z+\frac{1}{2}$; (ii) $-x+2, y+\frac{1}{2},-z+\frac{3}{2}$.

Data collection: SMART (Siemens, 1996); cell refinement: SMART and SAINT (Siemens, 1994); data reduction: XPREP (Siemens, 1994); $\operatorname{program}(\mathrm{s})$ used to solve structure: SHELXTL (Sheldrick, 2008); program(s) used to refine structure: SHELXTL; molecular graphics: $S H E L X T L$; software used to prepare material for publication: SHELXTL.

This study was supported by the National Science Foundation of China (C050102).

Supplementary data and figures for this paper are available from the IUCr electronic archives (Reference: BX2320).

\title{
References
}

Karapetyan, H. A. (2008a). Acta Cryst. E64, o1222.

Karapetyan, H. A. (2008b). Acta Cryst. E64, o943.

Sheldrick, G. M. (1996). SADABS. University of Göttingen, Germany.

Sheldrick, G. M. (2008). Acta Cryst. A64, 112-122.

Siemens (1994). SAINT. Siemens Analytical X-ray Instruments Inc., Madison, Wisconsin, USA.

Siemens (1996). SMART. Siemens Analytical X-ray Instruments Inc., Madison, Wisconsin, USA. 


\section{supporting information}

Acta Cryst. (2011). E67, o10 [https://doi.org/10.1107/S1600536810049676]

\section{2-Iminiumyl-1,3-diazepane-4-carboxylate}

\section{Feng Yang}

\section{S1. Comment}

The title compound (I) was hydrothermally synthesized from $L$-Arginine via an unusual annulation reaction. We report here a new annulation product derived from the linear arginine molecule. The title compound is the cyclic form of $L$ Arginine and this molecule is a zwitterion with the positive and negative charge residing in the guanidinium and carboxylate groups respectively. The conformation of 1,3-diazepane ring is close to twisted chair.One intramolecular and three intermolecular $\mathrm{N}-\mathrm{H} \cdots \mathrm{O}$ hydrogen bonds stabilize the molecular conformation and the crystal structure respectively (Fig. 2). The $\mathrm{C}-\mathrm{N}$ distances in the guanidinium group are obviously shorter than that of the normal $\mathrm{C}-\mathrm{N}$ single bond, indicating delocalized bond of the guanidinium group.

\section{S2. Experimental}

A mixture of $\mathrm{Cu}\left(\mathrm{ClO}_{4}\right)_{2} 6 \mathrm{H}_{2} \mathrm{O}(0.186 \mathrm{~g}, 0.5 \mathrm{mmol}), L$-Arginine $(0.087 \mathrm{~g}, 0.5 \mathrm{mmol})$ and water $(10 \mathrm{ml})$ was sealed in a 15 $\mathrm{ml}$ teflon-lined stainless steel reactor and heated to $423 \mathrm{~K}$ for $60 \mathrm{~h}$. Colorless crystals of (I) suitable for X-ray analysis were obtained.

\section{S3. Refinement}

All $\mathrm{H}$ atoms were placed at calculated positions, and refined with isotropic displacement parameters, using a riding model $\left[\mathrm{C}-\mathrm{H}=0.97 \AA\right.$ and $U_{\text {iso }}(\mathrm{H})=1.2 U_{\text {eq }}(\mathrm{C}), \mathrm{C}-\mathrm{H}=0.98 \AA$ and $U_{\text {iso }}(\mathrm{H})=1.5 U_{\text {eq }}(\mathrm{C}), \mathrm{N}-\mathrm{H}=0.86 \AA$ and $U_{\text {iso }}(\mathrm{H})=$ $\left.1.2 U_{\mathrm{eq}}(\mathrm{N})\right]$. 


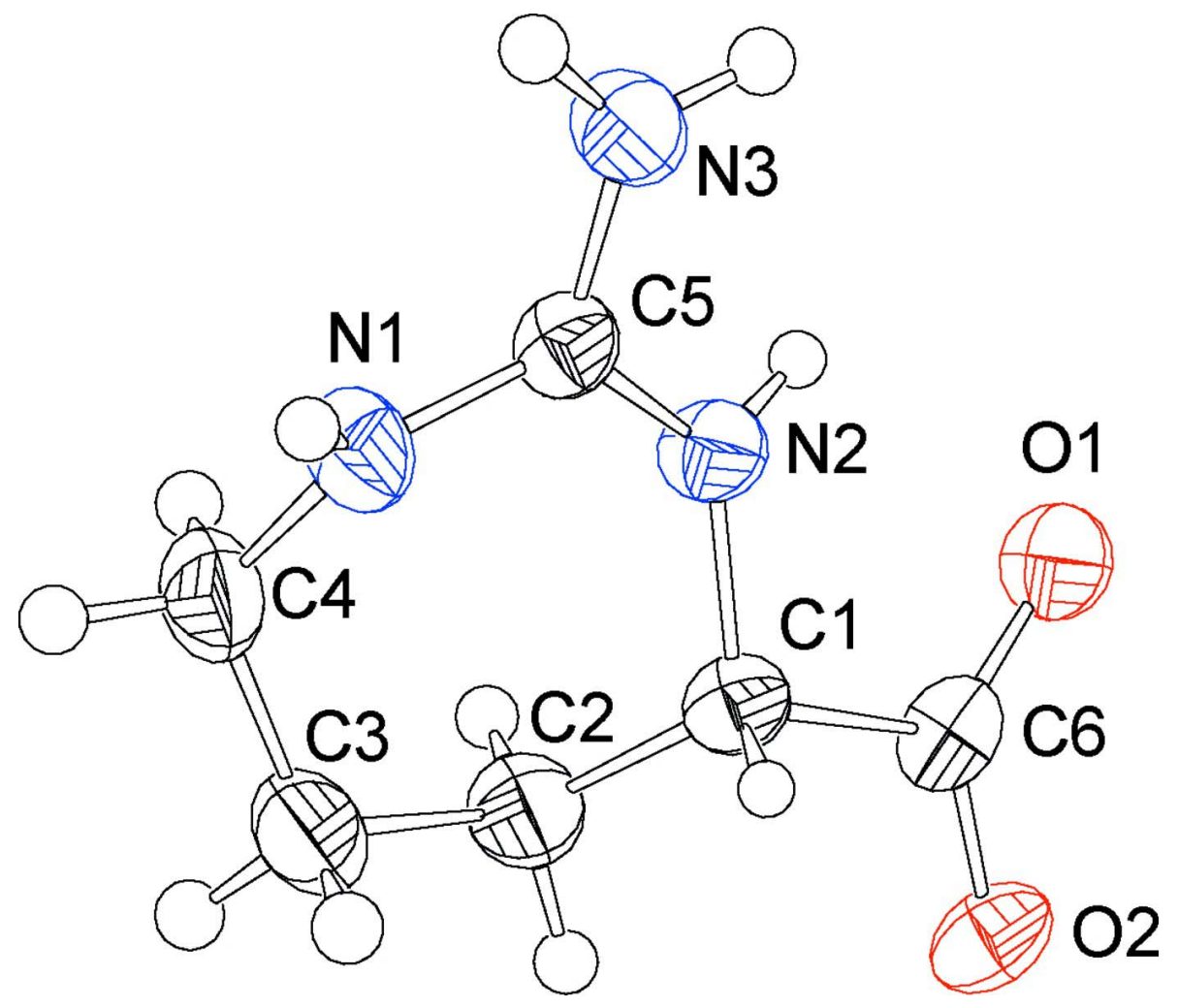

Figure 1

A view of (I) with $50 \%$ probability displacement ellipsoids; $\mathrm{H}$ atoms are shown as small spheres of arbitrary radii.

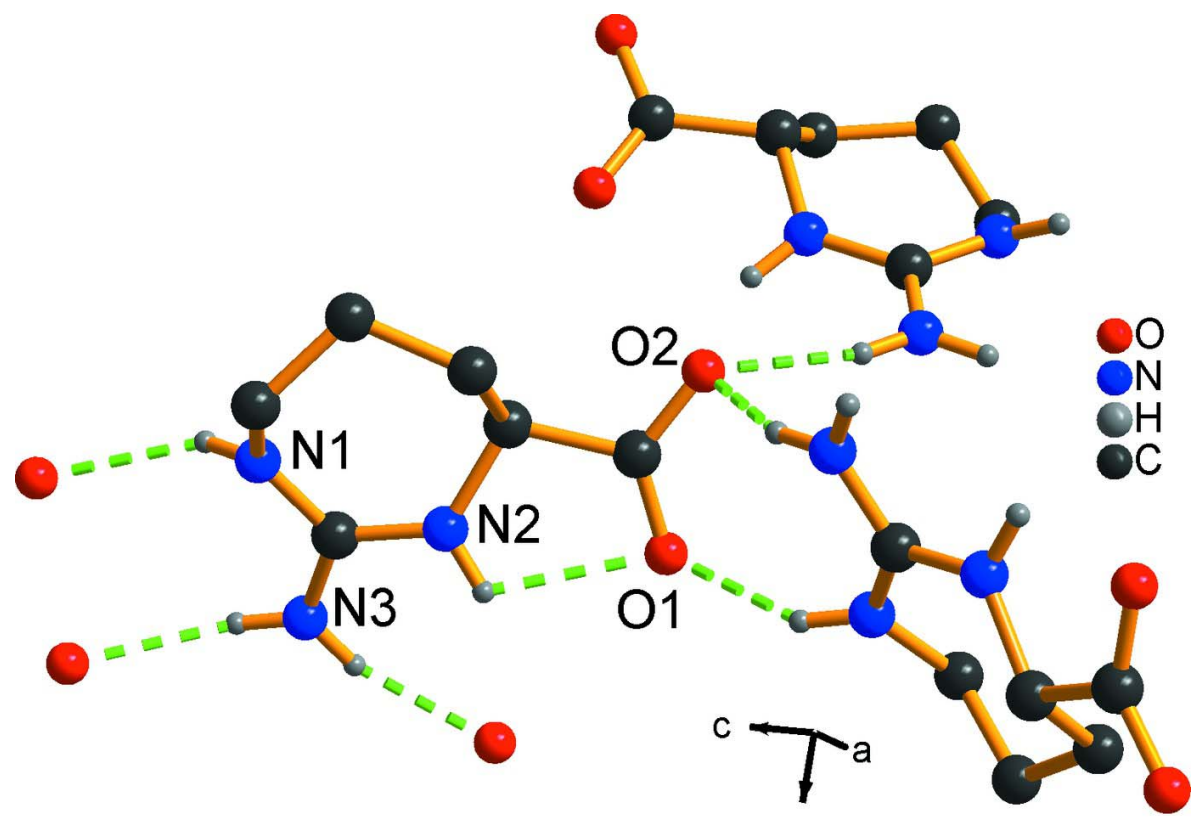

b

\section{Figure 2}

The hydrogen bonding interactions between the molecules. The H- atoms not involved in hydrogen bond are omitted for charity 
2-iminiumyl-1,3-diazepane-4-carboxylate

Crystal data

$\mathrm{C}_{6} \mathrm{H}_{11} \mathrm{~N}_{3} \mathrm{O}_{2}$

$M_{r}=157.18$

Orthorhombic, $P 2_{1} 2_{1} 2_{1}$

Hall symbol: P $2 \mathrm{ac} 2 \mathrm{ab}$

$a=6.1740$ (3) $\AA$

$b=8.7979(5) \AA$

$c=14.2036(7) \AA$

$V=771.51(7) \AA^{3}$

$Z=4$

$F(000)=336$

$D_{\mathrm{x}}=1.353 \mathrm{Mg} \mathrm{m}^{-3}$

Mo $K \alpha$ radiation, $\lambda=0.71073 \AA$

Cell parameters from 1112 reflections

$\theta=2.7-20.9^{\circ}$

$\mu=0.10 \mathrm{~mm}^{-1}$

$T=293 \mathrm{~K}$

Prism, colorless

$0.23 \times 0.15 \times 0.10 \mathrm{~mm}$

\section{Data collection}

Siemens SMART CCD area-detector diffractometer

Radiation source: fine-focus sealed tube

Graphite monochromator

phi and $\omega$ scans

Absorption correction: multi-scan

(SADABS; Sheldrick, 1996)

$T_{\min }=0.773, T_{\max }=1.000$

3426 measured reflections

834 independent reflections

694 reflections with $I>2 \sigma(I)$

$R_{\text {int }}=0.026$

$\theta_{\max }=25.1^{\circ}, \theta_{\min }=2.7^{\circ}$

$h=-7 \rightarrow 5$

$k=-9 \rightarrow 10$

$l=-16 \rightarrow 13$

Refinement

Refinement on $F^{2}$

Least-squares matrix: full

$R\left[F^{2}>2 \sigma\left(F^{2}\right)\right]=0.042$

$w R\left(F^{2}\right)=0.116$

$S=1.08$

834 reflections

100 parameters

0 restraints

Primary atom site location: structure-invariant direct methods

Secondary atom site location: difference Fourier map

Hydrogen site location: inferred from neighbouring sites

$\mathrm{H}$-atom parameters constrained

$w=1 /\left[\sigma^{2}\left(F_{\mathrm{o}}^{2}\right)+(0.0573 P)^{2}+0.2708 P\right]$

where $P=\left(F_{\mathrm{o}}^{2}+2 F_{\mathrm{c}}^{2}\right) / 3$

$(\Delta / \sigma)_{\max }=0.002$

$\Delta \rho_{\max }=0.25$ e $\AA^{-3}$

$\Delta \rho_{\min }=-0.23 \mathrm{e} \AA^{-3}$

Special details

Geometry. All e.s.d.'s (except the e.s.d. in the dihedral angle between two 1.s. planes) are estimated using the full covariance matrix. The cell e.s.d.'s are taken into account individually in the estimation of e.s.d.'s in distances, angles and torsion angles; correlations between e.s.d.'s in cell parameters are only used when they are defined by crystal symmetry. An approximate (isotropic) treatment of cell e.s.d.'s is used for estimating e.s.d.'s involving 1.s. planes.

Refinement. Refinement of $F^{2}$ against ALL reflections. The weighted $R$-factor $w R$ and goodness of fit $S$ are based on $F^{2}$, conventional $R$-factors $R$ are based on $F$, with $F$ set to zero for negative $F^{2}$. The threshold expression of $F^{2}>\sigma\left(F^{2}\right)$ is used only for calculating $R$-factors(gt) etc. and is not relevant to the choice of reflections for refinement. $R$-factors based on $F^{2}$ are statistically about twice as large as those based on $F$, and $R$ - factors based on ALL data will be even larger.

Fractional atomic coordinates and isotropic or equivalent isotropic displacement parameters $\left(\AA^{2}\right)$

\begin{tabular}{lllll}
\hline & $x$ & $y$ & $z$ & $U_{\text {iso }}{ }^{*} / U_{\text {eq }}$ \\
\hline O1 & $0.9643(4)$ & $0.4869(3)$ & $0.67888(15)$ & $0.0471(7)$ \\
O2 & $0.8529(4)$ & $0.2509(2)$ & $0.64723(15)$ & $0.0463(7)$ \\
N1 & $0.5689(4)$ & $0.4568(3)$ & $0.97693(19)$ & $0.0413(8)$ \\
H1A & 0.5874 & 0.4365 & 1.0356 & $0.050^{*}$ \\
N2 & $0.7752(5)$ & $0.5024(3)$ & $0.84226(17)$ & $0.0383(7)$
\end{tabular}




$\begin{array}{lllll}\mathrm{H} 2 \mathrm{~A} & 0.8317 & 0.5757 & 0.8105 & 0.046^{*} \\ \mathrm{~N} 3 & 0.8387(5) & 0.6355(3) & 0.9771(2) & 0.0492(8) \\ \mathrm{H} 3 \mathrm{~A} & 0.8106 & 0.6559 & 1.0350 & 0.059^{*} \\ \mathrm{H} 3 \mathrm{~B} & 0.9404 & 0.6835 & 0.9483 & 0.059^{*} \\ \mathrm{C} 1 & 0.7420(6) & 0.3583(4) & 0.7926(2) & 0.0352(8) \\ \mathrm{H} 1 \mathrm{~B} & 0.8059 & 0.2765 & 0.8303 & 0.042^{*} \\ \mathrm{C} 2 & 0.5046(7) & 0.3203(4) & 0.7748(2) & 0.0486(10) \\ \mathrm{H} 2 \mathrm{~B} & 0.4346 & 0.4071 & 0.7457 & 0.058^{*} \\ \mathrm{H} 2 \mathrm{C} & 0.4962 & 0.2359 & 0.7310 & 0.058^{*} \\ \mathrm{C} 3 & 0.3829(7) & 0.2787(4) & 0.8645(3) & 0.0558(11) \\ \mathrm{H} 3 \mathrm{C} & 0.2381 & 0.2455 & 0.8479 & 0.067^{*} \\ \mathrm{H} 3 \mathrm{D} & 0.4560 & 0.1940 & 0.8946 & 0.067^{*} \\ \mathrm{C} 4 & 0.3667(6) & 0.4072(4) & 0.9331(3) & 0.0510(10) \\ \mathrm{H} 4 \mathrm{~A} & 0.3033 & 0.4936 & 0.9008 & 0.061^{*} \\ \mathrm{H} 4 \mathrm{~B} & 0.2671 & 0.3775 & 0.9826 & 0.061^{*} \\ \mathrm{C} 5 & 0.7260(5) & 0.5309(4) & 0.9325(2) & 0.0348(8) \\ \mathrm{C} 6 & 0.8659(6) & 0.3684(4) & 0.6983(2) & 0.0372(8)\end{array}$

Atomic displacement parameters $\left(\AA^{2}\right)$

\begin{tabular}{lllllll}
\hline & $U^{11}$ & $U^{22}$ & $U^{33}$ & $U^{12}$ & $U^{13}$ & $U^{23}$ \\
\hline $\mathrm{O} 1$ & $0.0583(16)$ & $0.0522(14)$ & $0.0306(12)$ & $-0.0038(14)$ & $0.0092(11)$ & $0.0005(11)$ \\
$\mathrm{O} 2$ & $0.0625(17)$ & $0.0467(13)$ & $0.0299(11)$ & $0.0092(13)$ & $0.0014(13)$ & $-0.0071(10)$ \\
$\mathrm{N} 1$ & $0.0450(18)$ & $0.0527(17)$ & $0.0261(13)$ & $-0.0089(15)$ & $0.0052(13)$ & $0.0025(13)$ \\
$\mathrm{N} 2$ & $0.0500(18)$ & $0.0391(14)$ & $0.0257(13)$ & $-0.0051(14)$ & $0.0053(12)$ & $-0.0024(12)$ \\
$\mathrm{N} 3$ & $0.057(2)$ & $0.0567(18)$ & $0.0343(14)$ & $-0.0191(17)$ & $0.0114(15)$ & $-0.0135(14)$ \\
$\mathrm{C} 1$ & $0.043(2)$ & $0.0356(15)$ & $0.0264(16)$ & $0.0019(16)$ & $-0.0015(15)$ & $-0.0009(14)$ \\
$\mathrm{C} 2$ & $0.051(2)$ & $0.057(2)$ & $0.038(2)$ & $-0.008(2)$ & $0.0000(19)$ & $-0.0098(17)$ \\
$\mathrm{C} 3$ & $0.049(2)$ & $0.061(2)$ & $0.057(2)$ & $-0.017(2)$ & $0.005(2)$ & $-0.005(2)$ \\
$\mathrm{C} 4$ & $0.042(2)$ & $0.063(2)$ & $0.047(2)$ & $-0.0080(19)$ & $0.009(2)$ & $-0.0011(18)$ \\
$\mathrm{C} 5$ & $0.0395(19)$ & $0.0370(16)$ & $0.0279(15)$ & $0.0017(17)$ & $0.0017(15)$ & $0.0010(14)$ \\
C6 & $0.040(2)$ & $0.046(2)$ & $0.0256(16)$ & $0.0110(18)$ & $-0.0035(16)$ & $0.0018(15)$ \\
& & & & & &
\end{tabular}

Geometric parameters ( $\left.\AA,{ }^{\circ}\right)$

\begin{tabular}{llll}
\hline $\mathrm{O} 1-\mathrm{C} 6$ & $1.238(4)$ & $\mathrm{C} 1-\mathrm{C} 2$ & $1.524(6)$ \\
$\mathrm{O} 2-\mathrm{C} 6$ & $1.265(4)$ & $\mathrm{C} 1-\mathrm{C} 6$ & $1.545(4)$ \\
$\mathrm{N} 1-\mathrm{C} 5$ & $1.329(4)$ & $\mathrm{C} 1-\mathrm{H} 1 \mathrm{~B}$ & 0.9800 \\
$\mathrm{~N} 1-\mathrm{C} 4$ & $1.462(4)$ & $\mathrm{C} 2-\mathrm{C} 3$ & $1.523(5)$ \\
$\mathrm{N} 1-\mathrm{H} 1 \mathrm{~A}$ & 0.8600 & $\mathrm{C} 2-\mathrm{H} 2 \mathrm{~B}$ & 0.9700 \\
$\mathrm{~N} 2-\mathrm{C} 5$ & $1.340(4)$ & $\mathrm{C} 2-\mathrm{H} 2 \mathrm{C}$ & 0.9700 \\
$\mathrm{~N} 2-\mathrm{C} 1$ & $1.465(4)$ & $\mathrm{C} 3-\mathrm{C} 4$ & $1.496(5)$ \\
$\mathrm{N} 2-\mathrm{H} 2 \mathrm{~A}$ & 0.8600 & $\mathrm{C} 3-\mathrm{H} 3 \mathrm{C}$ & 0.9700 \\
$\mathrm{~N} 3-\mathrm{C} 5$ & $1.317(4)$ & $\mathrm{C} 3-\mathrm{H} 3 \mathrm{D}$ & 0.9700 \\
$\mathrm{~N} 3-\mathrm{H} 3 \mathrm{~A}$ & 0.8600 & $\mathrm{C} 4-\mathrm{H} 4 \mathrm{~A}$ & 0.9700 \\
$\mathrm{~N} 3-\mathrm{H} 3 \mathrm{~B}$ & 0.8600 & $\mathrm{C} 4-\mathrm{H} 4 \mathrm{~B}$ & 0.9700 \\
& & & \\
$\mathrm{C} 5-\mathrm{N} 1-\mathrm{C} 4$ & $124.6(3)$ & $\mathrm{H} 2 \mathrm{~B}-\mathrm{C} 2-\mathrm{H} 2 \mathrm{C}$ & 107.8
\end{tabular}




$\begin{array}{llll}\mathrm{C} 5-\mathrm{N} 1-\mathrm{H} 1 \mathrm{~A} & 117.7 & \mathrm{C} 4-\mathrm{C} 3-\mathrm{C} 2 & 113.3(3) \\ \mathrm{C} 4-\mathrm{N} 1-\mathrm{H} 1 \mathrm{~A} & 117.7 & \mathrm{C} 4-\mathrm{C} 3-\mathrm{H} 3 \mathrm{C} & 108.9 \\ \mathrm{C} 5-\mathrm{N} 2-\mathrm{C} 1 & 126.1(3) & \mathrm{C} 2-\mathrm{C} 3-\mathrm{H} 3 \mathrm{C} & 108.9 \\ \mathrm{C} 5-\mathrm{N} 2-\mathrm{H} 2 \mathrm{~A} & 116.9 & \mathrm{C} 4-\mathrm{C} 3-\mathrm{H} 3 \mathrm{D} & 108.9 \\ \mathrm{C} 1-\mathrm{N} 2-\mathrm{H} 2 \mathrm{~A} & 116.9 & \mathrm{C} 2-\mathrm{C} 3-\mathrm{H} 3 \mathrm{D} & 108.9 \\ \mathrm{C} 5-\mathrm{N} 3-\mathrm{H} 3 \mathrm{~A} & 120.0 & \mathrm{H} 3 \mathrm{C}-\mathrm{C} 3-\mathrm{H} 3 \mathrm{D} & 107.7 \\ \mathrm{C} 5-\mathrm{N} 3-\mathrm{H} 3 \mathrm{~B} & 120.0 & \mathrm{~N} 1-\mathrm{C} 4-\mathrm{C} 3 & 116.5(3) \\ \mathrm{H} 3 \mathrm{~A}-\mathrm{N} 3-\mathrm{H} 3 \mathrm{~B} & 120.0 & \mathrm{~N} 1-\mathrm{C} 4-\mathrm{H} 4 \mathrm{~A} & 108.2 \\ \mathrm{~N} 2-\mathrm{C} 1-\mathrm{C} 2 & 113.9(3) & \mathrm{C} 3-\mathrm{C} 4-\mathrm{H} 4 \mathrm{~A} & 108.2 \\ \mathrm{~N} 2-\mathrm{C} 1-\mathrm{C} 6 & 107.3(3) & \mathrm{N} 1-\mathrm{C} 4-\mathrm{H} 4 \mathrm{~B} & 108.2 \\ \mathrm{C} 2-\mathrm{C} 1-\mathrm{C} 6 & 110.2(3) & \mathrm{C} 3-\mathrm{C} 4-\mathrm{H} 4 \mathrm{~B} & 108.2 \\ \mathrm{~N} 2-\mathrm{C} 1-\mathrm{H} 1 \mathrm{~B} & 108.4 & \mathrm{H} 4 \mathrm{~A}-\mathrm{C} 4-\mathrm{H} 4 \mathrm{~B} & 120.3 \\ \mathrm{C} 2-\mathrm{C} 1-\mathrm{H} 1 \mathrm{~B} & 108.4 & \mathrm{~N} 3-\mathrm{C} 5-\mathrm{N} 1 & 118.1(3) \\ \mathrm{C} 6-\mathrm{C} 1-\mathrm{H} 1 \mathrm{~B} & 108.4 & \mathrm{~N} 3-\mathrm{C} 5-\mathrm{N} 2 & 121.9(3) \\ \mathrm{C} 3-\mathrm{C} 2-\mathrm{C} 1 & 112.8(3) & \mathrm{N} 1-\mathrm{C} 5-\mathrm{N} 2 & 126.2(3) \\ \mathrm{C} 3-\mathrm{C} 2-\mathrm{H} 2 \mathrm{~B} & 109.0 & \mathrm{O} 1-\mathrm{C} 6-\mathrm{O} 2 & 119.0(3) \\ \mathrm{C} 1-\mathrm{C} 2-\mathrm{H} 2 \mathrm{~B} & 109.0 & \mathrm{O} 1-\mathrm{C} 6-\mathrm{C} 1 & 114.8(3) \\ \mathrm{C} 3-\mathrm{C} 2-\mathrm{H} 2 \mathrm{C} & 109.0 & \mathrm{O} 2-\mathrm{C} 6-\mathrm{C} 1 & \\ \mathrm{C} 1-\mathrm{C} 2-\mathrm{H} 2 \mathrm{C} & 109.0 & & \end{array}$

Hydrogen-bond geometry $\left(A,{ }^{\circ}\right)$

\begin{tabular}{lllll}
\hline$D-\mathrm{H} \cdots A$ & $D-\mathrm{H}$ & $\mathrm{H} \cdots A$ & $D \cdots A$ & $D-\mathrm{H} \cdots A$ \\
\hline $\mathrm{N} 1-\mathrm{H} 1 A \cdots \mathrm{O} 1^{\mathrm{i}}$ & 0.86 & 2.17 & $2.918(3)$ & 146 \\
$\mathrm{~N} 3-\mathrm{H} 3 A \cdots \mathrm{O} 2^{\mathrm{i}}$ & 0.86 & 2.06 & $2.870(4)$ & 157 \\
$\mathrm{~N} 3-\mathrm{H} 3 B \cdots \mathrm{O} 2^{2 i}$ & 0.86 & 1.95 & $2.788(4)$ & 163 \\
$\mathrm{~N} 2-\mathrm{H} 2 A \cdots \mathrm{O} 1$ & 0.86 & 2.19 & $2.601(3)$ & 109 \\
\hline
\end{tabular}

Symmetry codes: (i) $-x+3 / 2,-y+1, z+1 / 2$; (ii) $-x+2, y+1 / 2,-z+3 / 2$. 